International Journal of Modern Physics A,

(C) World Scientific Publishing Company

\title{
THE EXACT COSMOLOGICAL SOLUTION TO THE DYNAMICAL EQUATIONS FOR THE BIANCHI-IX MODEL
}

\author{
V.A. SAVCHENKO T.P. SHESTAKOVAl G.M. VERESHKOV \\ Department of theoretical physics, Rostov State University \\ Sorge str. 5, Rostov-on-Don 344090, Russia
}

Received 5 April 1999

\begin{abstract}
Quantum geometrodynamics in extended phase space describes phenomenologically the integrated system "a physical object + observation means (a gravitational vacuum condensate)". The central place in this version of QGD belongs to the Schrödinger equation for a wave function of the Universe. An exact solution to the "conditionally-classical" set of equations in extended phase space for the Bianchi-IX model and the appropriate solution to the Schrödinger equation are considered. The physical adequacy of the obtained solutions to existing concepts about possible cosmological scenarios is demonstrated. The gravitational vacuum condensate is shown to be a cosmological evolution factor.
\end{abstract}

\section{Introduction}

In the previous paper we studied quantum geometrodynamics (QGD) of the Bianchi-IX model in the framework of extended phase space (EPS) approach elaborated by Batalin, Fradkin and Vilkovisky (BFV) the BFV approach one singles out a BRST-invariant sector which is supposed to coincide with a gauge-invariant one. This scheme was realized in the workst it has a manifest physical interpretation in the case of S-matrix theory. However, in quantum cosmology appropriate mathematical operations are just formal and the question arises, whether these operations are mathematically and physically justified. This question has been explored in our papert. Our main result consisted in the demonstration that in a closed universe without asymptotical states BRST-invariance is not equivalent to gauge invariance. A mathematical indication to the nonequivalence of gauge and BRST invariance is given by the well-known parametrization noninvariance of the Wheeler - DeWitt (WDW) equation. As we have shown in the framework of the BFV approach, the latter is an ill-hidden gauge noninvariance. For this reason singling out a BRST-invariant sector is not motivated in QGD and is not used in our approach. This circumstance distinguished our version of QGD from other works on quantum geometrodynamics. A physical ground for our approach consists in the impossibility to remove an observer from a

*e-mail: savchenko@phys.rnd.runnet.ru

$\dagger_{\text {e }}$-mail: stp@phys.rnd.runnet.ru 
closed universe, and, as a consequence, the necessity to take into account his affecting physical processes. The lack of gauge symmetry, or, more precisely, breaking down this symmetry when considering observation means (OM) as a part of an integrated system in our version of QGD is described by eigenvalues of gravitational super-Hamiltonian; we refer to the latters as energy levels of gravitation vacuum condensate (GVC).

It is worth emphasizing that in a gauge-invariant approach to QGD it is assumed that the information about the reduction of a wave packet in the process of evolution of the Universe is contained in boundary conditions -10 . In contrast to the conventional approach, our version of QGD admits the Copenhagen interpretation and aims at describing the integrated system "a physical object (gravitational field) + observation means (gravitational vacuum condensate)".

Approaches of quantum cosmology are traditionally tested and_developed for the Bianchi-IX model and its particular case - the isotropic mode11-15. As it is known, the Bianchi-IX model can be represented as an isotropic space where two transversal nonlinear gravitational waves are excited. So, in papers based on the conventional Wheeler - DeWitt QGD (or in those where the BRST-invariant sector in EPS is singled out) observables include 3-space volume and amplitudes of gravitational waves. The convential approach is developing applying new consepts such as supergavity and superstring, $16-21$. However, the interpretation of the Wheeler De Witt theory is not changing: the information about the past of the Universe as well as its future is contained in boundary conditions. In our modification of QGD a new feature appears - the gravitational vacuum condensate with its degrees of freedom. Wave functions of the integrated system are represented by normalized wave packets; we suppose that their structure is determined while the Universe creating from "Nothing". The influence of observation means on the evolution of the integrated system in the proposed version of QGD is described by transformation of the form of a wave packet.

The wave functions satisfy the dynamical Schrödinger equation (SE). For the Bianchi-IX model the normalized in the EPS general solution (GS) to the gaugenoninvariant SE reads

$$
\Psi\left(Q^{a}, Q^{0}, \theta, \bar{\theta} ; t\right)=\int \Psi_{k}\left(Q^{a}\right) \exp \left(-i E_{k} t\right)(\bar{\theta}+i \theta) \delta\left(Q^{0}-f\left(Q^{a}\right)-k\right) d E_{k} d k,
$$

where $\Psi_{k}\left(Q^{a}\right)$ is a solution to the stationary equation

$$
H_{k}^{0} \Psi_{k}\left(Q^{a}\right)=E_{k} \Psi_{k}\left(Q^{a}\right),
$$

$\theta, \bar{\theta}$ are the ghosts, $Q^{0}$ and $Q^{a}$ are the gauge and physical variables respectively, specification of which see below in Sec. 2 .

The norm integral for the WF (11) over the full set of variables $Q, \theta, \bar{\theta}$ results in

$$
\int \Psi_{k}^{*}\left(Q^{a}, t\right) \Psi_{k}\left(Q^{a}, t\right) M_{k}\left(Q^{a}\right) d k \prod_{a} d Q^{a},
$$


so the GS to the SE, under the condition the $\Psi_{k}\left(Q^{a}, t\right)$ to be a sufficiently narrow packet over $k$, is normalizable with respect to the gauge variable, as well as to the ghosts and the physical variables.

The theory does not control the WF dependence on the parameter $k$ which in the classical dynamics determines an initial clock setting, and this additional degree of freedom has to be referred to an observer.

Investigation of the particular BRST-invariant solution has shown that it really satisfies the WDW equation but cannot be normalized. On the other hand, as we have already mentioned above, the well known parametrization noninvariance of the WDW equations is shown to be the ill-hidden gauge noninvariance. The latter means that the WDW theory does not achieve its object to give a gauge-invariant description of the Universe.

By this reason and because of the loss of probability interpretation we do not see any ground to be guided by the WDW theory, and explore the possibilities of the gauge-noninvariant QGD version.

The factored part of the GS (1) - $\delta$-function and ghosts - represents the OM described by the gauge-fixing term in the Lagrangian and by the appropriate "energymomentum tensor" (quasi-EMT). The latter corresponds to the continuous medium that fills the whole space and can be called "gravitational vacuum condensate"; it is quantitatively fixed by an eigenvalue $E_{k}$ of the Hamiltonian $H_{k}^{0}$. The structure of the GS shows a GVC to be an important factor of the global evolution. The purpose of this paper is to investigate this effect on the base of the exact solution to the $\mathrm{SE}$ for the simplified model with the frozen degree of freedom $Q^{3}$, in a simple gauge.

In Sec. . all the necessary notations and the general equations for the Bianchi-IX model are given; in Sec. . the exact conditionally-classical solution for the simple case is considered. We shall show that GVC can play a decisive role in forming non-trivial cosmological scenarios. In Sec.. the exact solution to the SE is considered, we analyse the formation and properties of the wave packet representing the evolving universe. In Sec. . the transition to the semiclassical solution is considered and it is shown how the quantum cosmological effects become negligible in the semiclassical region; here various cosmological scenarios depending on the GVC and the homogeneous scalar field are considered. In Sec. . the concept of time in the gauge-noninvariant QGD is discussed.

\section{The General Model Equations}

The Bianchi-IX 4-interval is given by

$$
\begin{gathered}
d s^{2}=N^{2}(t) d t^{2}-\eta_{a b}(t) e_{i}^{a} e_{k}^{b} d x^{i} d x^{k} ; \\
\eta_{a b}(t)=\operatorname{diag}\left(a^{2}(t), b^{2}(t), c^{2}(t)\right),
\end{gathered}
$$




$$
\begin{aligned}
e_{i}^{1} & =\left(\sin x^{3},-\cos x^{3} \sin x^{1}, 0\right), \\
e_{i}^{2} & =\left(\cos x^{3}, \sin x^{3} \sin x^{1}, 0\right), \\
e_{i}^{3} & =\left(0, \cos x^{1}, 1\right) . \\
a=\frac{1}{2} \exp \left[\frac { 1 } { 2 } \left(Q^{1}+Q^{2}+\right.\right. & \left.\left.\sqrt{3} Q^{3}\right)\right] ; b=\frac{1}{2} \exp \left[\frac{1}{2}\left(Q^{1}+Q^{2}-\sqrt{3} Q^{3}\right)\right] ; \\
c & =\frac{1}{2} \exp \left(\frac{1}{2} Q^{1}-Q^{3}\right) .
\end{aligned}
$$

The model is assumed to include an arbitrary number $K$ of real homogeneous scalar fields $Q^{4}, \ldots, Q^{K+3}$ with some potential $U_{s}$. The gauge coordinate $Q^{0}$ is defined by an arbitrary parametrization function

$$
\zeta\left(Q^{0}, Q^{1}, \ldots, Q^{K+3}\right)=\ln \left[\frac{1}{N} \exp \left(\frac{3}{2} Q^{1}\right)\right],
$$

and gauges are supposed to be not depending on time,

$$
Q^{0}=f\left(Q^{1}, \ldots\right)+k, k=\text { const. }
$$

The SE reads

$$
i \frac{\partial \Psi\left(Q^{0}, Q^{1}, \ldots, \theta, \bar{\theta} ; t\right)}{\partial t}=H \Psi\left(Q^{0}, Q^{1}, \ldots, \theta, \bar{\theta} ; t\right),
$$

where $\Psi\left(Q^{\alpha}, \theta, \bar{\theta} ; t\right)$ is a universe $\mathrm{WF}(\alpha=0, a ; a=1, \ldots, K+3), \theta, \bar{\theta}$, are the ghosts,

$$
H=-i \zeta, 0 \frac{\partial}{\partial \theta} \frac{\partial}{\partial \bar{\theta}}-\frac{1}{2 M} \frac{\partial}{\partial Q^{\alpha}} G^{\alpha \beta} \frac{\partial}{\partial Q^{\beta}}+\mathrm{e}^{-\zeta}(U-V),
$$

$\zeta_{, 0}=\partial \zeta\left(Q^{\alpha}\right) / \partial Q^{0}$, probability measure

$$
\begin{aligned}
& M=\text { const } \cdot \zeta_{, 0} \exp \left(\frac{K+3}{2} \zeta\right) \\
& U(Q)=\mathrm{e}^{2 Q^{1}} U_{g}\left(Q^{2}, Q^{3}\right)+\mathrm{e}^{3 Q^{1}} U_{s}\left(Q^{4}, \ldots\right), \\
& U_{g}\left(Q^{2}, Q^{3}\right)=\frac{2}{3}\left\{\exp \left[2\left(Q^{2}+\sqrt{3} Q^{3}\right)\right]+\exp \left[2\left(Q^{2}-\sqrt{3} Q^{3}\right)\right]+\exp (-4 \chi)\right. \\
& \left.-2 \exp \left[-\left(Q^{2}+\sqrt{3} Q^{3}\right)\right]-2 \exp \left(-Q^{2}+\sqrt{3} Q^{3}\right)-2 \exp \left(2 Q^{2}\right)\right\} \\
& V=-\frac{3}{12} \frac{(\zeta, 0)^{a}\left(\zeta_{, 0}\right)_{a}}{\zeta,,^{2}}+\frac{(\zeta, 0)_{a}^{a}}{3 \zeta, 0}+\frac{K+1}{6 \zeta, 0} \zeta_{a}(\zeta, 0)^{a} \\
& +\frac{1}{24}\left(K^{2}+3 K+14\right) \zeta_{a} \zeta^{a}+\frac{K+2}{6} \zeta_{a}^{a}, \\
& \zeta_{a}=\partial \zeta / \partial Q^{a}+f,_{a} \partial \zeta / \partial Q^{0}, \\
& G^{\alpha \beta}=M \mathrm{e}^{-\zeta}\left(\begin{array}{cc}
f_{,{ }_{a} f^{, a}} & f^{, a} \\
f^{, a} & \gamma^{a b}
\end{array}\right) .
\end{aligned}
$$


To make the analysis more visual, we begin with considering the conditionallyclassical solution to the Einstein equations supplemented with the quasi-EMT of the ghosts and the GVC. In common case this set of equations, yielded by the "classical version" of the Hamiltonian (8), reads

$$
\begin{aligned}
& \left(\mathrm{e}^{\zeta} \dot{Q}_{a}\right)^{\cdot}+\mathrm{e}^{-\zeta} U_{,_{a}}-\dot{\lambda} f_{,_{a}}-\frac{1}{2} \zeta_{, a} \mathrm{e}^{\zeta} \dot{Q}^{b} \dot{Q}_{b}-\zeta_{, a} \mathrm{e}^{\zeta} U+\frac{i \zeta_{, 0}, a}{\zeta_{, 0}^{2}} \dot{\bar{\theta}} \dot{\theta}=0,( \\
& \frac{1}{2} \zeta, 0_{0} \mathrm{e}^{\zeta} \dot{Q}^{a} \dot{Q}_{a}+\zeta_{, 0} \mathrm{e}^{-\zeta} U-\frac{i \zeta, 0_{0},}{\zeta_{0}^{2}} \dot{\bar{\theta}} \dot{\theta}-\dot{\lambda}=0, \\
& \dot{Q}^{0}-f_{, a} \dot{Q}^{a}=0 \\
& \left(\zeta,{ }_{0}^{-1} \dot{\theta}\right)=0 \\
& \left(\zeta,,_{0}^{-1} \dot{\bar{\theta}}\right)=0
\end{aligned}
$$

where $\lambda=\pi+\dot{\bar{\theta}} \theta, \pi$ is the Lagrange multiple fixing the gauge (14), coupled to the integral of motion describing the GVC:

$$
(\zeta, 0)_{k}^{-1} \dot{\lambda}=E_{k}
$$

\section{The Conditionally-Classical Exact Solution}

Taking the parametrization and the gauge

$$
\zeta=Q^{0}=k
$$

$(k=$ const), one can obtain an exact particular solution to Eqs. (12) - (16) with $Q^{3}=0$. The existence of this solution gives the formal opportunity to consider the model without this degree of freedom.

Under the condition (17) the ghost variables vanish from Eqs. (12) - (14), and the latter form a closed set concerning the physical variables; the state equation of the GVC becomes extremely hard,

$$
\begin{gathered}
p=\varepsilon=-\frac{\dot{\lambda}}{2 \pi^{2}} \exp \left(k-3 Q^{1}\right), \\
\dot{\lambda}=E .
\end{gathered}
$$

Note that conditionality of the classical approach shows here in the ghost presence in the integral of motion (19)

$$
\dot{\lambda}=\dot{\pi}-\dot{\bar{\theta}} \dot{\theta}
$$

i.e. the forms made of the Grassmannian variables appear as parameters of the theory.

Let us turn to the case of a single massless linear scalar field $\phi=Q^{4}$ and put

$$
U_{s}(\phi)=0,
$$


The Exact Cosmological Solution to the Dynamical Equations for the Bianchi IX Model

in $(10)$. Now we have the simple equation for $\phi$

$$
\ddot{\phi}=0 \text {, }
$$

thus,

$$
\dot{\phi}=C_{s}=\text { const. }
$$

The scalar field behaves as a medium with positive energy density and with an extremely hard equation of state

$$
p_{(s c a l)}=\varepsilon_{(s c a l)} \propto \exp \left(-3 Q^{1}\right) \dot{\phi}^{2}=C_{s}^{2} \exp \left(-3 Q^{1}\right)
$$

like that of the GVC (18).

As one can see, in the present model the Universe is filled with the two-component medium described by the parameters $E$ and $C_{s}$. Below we will show that relation between the two parameters essentially affects cosmological evolution at the quantum stage of the Universe existence as well as at the semiclassical one. Here is the difference between our consideration and the usual investigation of the Bianchi-IX model in general relativity.

The equations for $Q^{1}, Q^{2}$ take the form:

$$
\begin{aligned}
& \ddot{Q}^{1}-\frac{4}{3}\left[\exp \left(2 Q^{1}-4 Q^{2}\right)-4 \exp \left(2 Q^{1}-Q^{2}\right)\right]=0, \\
& \ddot{Q}^{2}-\frac{4}{3}\left[2 \exp \left(2 Q^{1}-4 Q^{2}\right)-2 \exp \left(2 Q^{1}-Q^{2}\right)\right]=0 .
\end{aligned}
$$

Integration is simplified with the substitution

$$
z_{1}=2 Q^{1}-4 Q^{2}, \quad z_{2}=2 Q^{1}-Q^{2}
$$

after replacing

$$
t \rightarrow \mathrm{e}^{-k} t
$$

the solution is available in the form

$$
\exp \left(\frac{z_{2}}{2}\right)=\frac{\alpha}{\cosh \left[2 \alpha\left(t-t_{0}\right)\right]}, \quad \exp \left(\frac{z_{1}}{2}\right)=\frac{\beta}{\cosh \left[2 \beta\left(t-t_{1}\right)\right]}
$$

where $\alpha, \beta, t_{0}, t_{1}$ are the integration constants. Without loss of generality, by shifting zero time one can put $t_{1}=0$. For the metric $(3)-(\sqrt{4})$ one finds:

$$
\begin{gathered}
a^{2}=b^{2}=\frac{1}{4} \exp \left(z_{2}-\frac{1}{2} z_{1}\right)=\frac{a^{2} \cosh (2 \beta t)}{4 \beta \cosh ^{2}\left[2 \alpha\left(t-t_{0}\right)\right]} \\
c^{2}=\frac{1}{4} \exp \left(\frac{1}{2} z_{1}\right)=\frac{\beta}{4 \cosh (2 \beta t)} .
\end{gathered}
$$

From the constraint equation (13) with $Q^{3}=0$, and (20) it follows:

$$
\frac{1}{24}\left(\dot{z}_{1}^{2}-4 \dot{z}_{2}^{2}\right)+\frac{2}{3}\left[\exp \left(z_{1}\right)-4 \exp \left(z_{2}\right)\right]=E_{k}-\frac{1}{2} C_{s}^{2},
$$


where $E_{k}=\mathrm{e}^{k} E$; hence, in turn,

$$
\alpha^{2}-\frac{1}{4} \beta^{2}=-\frac{3}{8}\left(E_{k}-\frac{1}{2} C_{s}^{2}\right)
$$

So, the dynamics of the model depends qualitatively on a relation between $C_{s}$ and $E_{k}$. Various cosmological effects of this dependence will be discussed in Sec. 5., after considering the quantum version of the exact solution.

\section{The Exact Solution to the Schrödinger Equation}

The task of constructing WF (1) is reduced to searching for a solution to stationary Eq. (2) for the physical part of the WF under the parametrization-and-gauge condition (17). This equation reads

$$
-\frac{1}{2} \frac{\partial^{2} \Psi_{k}}{\partial Q_{a} \partial Q^{a}}+U\left(Q^{a}\right) \Psi_{k}\left(Q^{a}\right)=E_{k} \Psi_{k}\left(Q^{a}\right),
$$

$a=(1,2,4)$. Substitution of (21) enables to separate the variables in the equation, and it can be written in the following manner

$$
\left(6 \hat{L}_{1}-\frac{3}{2} \hat{L}_{2}+\frac{1}{2} \hat{L}_{3}-k E\right) \Psi_{k}\left(z_{1}, z_{2}, \phi\right)=0,
$$

where

$$
\begin{aligned}
& \hat{L}_{1}=-\frac{\partial^{2}}{\partial z_{1}^{2}}+\frac{1}{9} \exp \left(z_{1}\right), \\
& \hat{L}_{2}=-\frac{\partial^{2}}{\partial z_{2}^{2}}+\frac{16}{9} \exp \left(z_{2}\right), \\
& \hat{L}_{3}=-\frac{\partial^{2}}{\partial \phi^{2}} .
\end{aligned}
$$

The eigenfunctions of the operators $\hat{L}_{1}, \hat{L}_{2}$ appropriate to the positive eigenvalues $\nu_{1}^{2} / 4, \nu_{2}^{2} / 4$ are the modified Bessel functions with an imaginary index,

$$
\psi_{\nu}(z)=\frac{1}{\sqrt{2 \pi \Gamma(i \nu)}} K_{i \nu}\left[A \exp \left(\frac{z}{2}\right)\right] ; \quad\left(A_{1} ; A_{2}\right)=\left(\frac{2}{3} ; \frac{8}{3}\right) .
$$

So far as

$$
\exp \left(\frac{z_{1}}{2}\right)=4 c^{2} ; \exp \left(\frac{z_{2}}{2}\right)=4 a c
$$

the quantum number $\nu_{1}$ determines probability distribution for the scale $c$, and so does the quantum number $\nu_{2}$ for the scale $a=b$ at a given $c$ value. Note, that for any $\nu$ there exists a semiclassical solution to the problem,

$$
\begin{aligned}
\psi_{\nu}(z) & =\frac{1}{\sqrt{2} \Gamma(i \nu) \exp \left(\frac{\pi \nu}{2}\right)\left(\frac{\nu^{2}}{4}-\frac{A^{2}}{4} \exp (z)\right)^{\frac{1}{4}}} \cos \left[2 \left(\sqrt{\frac{\nu^{2}}{4}-\frac{A^{2}}{4} \exp (z)}\right.\right. \\
& \left.\left.-\frac{\nu}{2} \operatorname{Artanh} \sqrt{1-\frac{A^{2}}{\nu^{2}} \exp (z)}\right)+\frac{\pi}{4}\right]
\end{aligned}
$$


$z<z_{\nu}$

$$
\begin{aligned}
\psi_{\nu}(z) & =\frac{1}{2 \sqrt{2} \Gamma(i \nu) \exp \left(\frac{\pi \nu}{2}\right)\left(\frac{A^{2}}{4} \exp (z)-\frac{\nu^{2}}{4}\right)^{\frac{1}{4}}} \exp \left[-2\left(\sqrt{\frac{A^{2}}{4} \exp (z)-\frac{\nu^{2}}{4}}\right.\right. \\
& \left.\left.-\frac{\nu}{2} \arctan \sqrt{\frac{A^{2}}{\nu^{2}} \exp (z)-1}\right)\right]
\end{aligned}
$$

$z>z_{\nu}, z_{\nu}=\ln \left(\nu^{2} / A^{2}\right)$ being the classical turning-point.

The operator $\hat{L}_{3}$ eigenfunctions are the plane waves

$$
\psi_{\varrho}(\phi)=\frac{1}{\sqrt{2 \pi}} \exp (i \varrho \phi)
$$

that is in agreement with the classical solution (20).

The GS to Eq. (24) for a given value of the parameter $E_{k}$, describing a GVC state, is a superposition

$$
\begin{aligned}
\Psi_{E_{k}}\left(z_{1}, z_{2}, \phi\right) & =\iint_{-\infty}^{\infty} \int \varrho d \nu_{1} d \nu_{2} c_{1}\left(\nu_{1}, \nu_{2}, \varrho\right) \psi_{\nu_{1}}\left(z_{1}\right) \psi_{\nu_{2}}\left(z_{2}\right) \psi_{\varrho}(\phi) \\
& \times \delta\left(\frac{3}{2} \nu_{1}^{2}-\frac{3}{8} \nu_{2}^{2}+\frac{1}{2} \varrho^{2}-E_{k}\right) .
\end{aligned}
$$

However, the stationary states, the wave functions (25) correspond to, are not physical, being unnormalizable because of continuity of the $E_{k}$ value spectrum. A time-dependent wave packet fits a physical state:

$$
\Psi_{k}\left(z_{1}, z_{2}, \phi, t\right)=\int_{-\infty}^{\infty} d E_{k} c_{2}\left(E_{k}\right) \Psi_{E_{k}}\left(z_{1}, z_{2}, \phi\right) \exp \left[-i E_{k}\left(t-t_{0}\right)\right] .
$$

Note, that in the expressions (25), (26) the quantity $E_{k}$ appears as a controlling parameter providing, via the $\delta$-function, correlation of the quantum numbers $\nu_{1}, \nu_{2}, \varrho$, and, by that, a probability distribution of the space scales at the quantum stage of the Universe evolution.

\section{The Semiclassical Regime and Cosmological Effects}

One can obtain the classical evolution law by computing the operators $\exp \left(z_{1} / 2\right)$, $\exp \left(z_{2} / 2\right)$ mean values over the packet (26). The matrix elements will be required for that, 


$$
\begin{aligned}
& \int_{-\infty}^{\infty} d z \exp \left(\frac{z}{2}\right) \psi_{\mu}^{*}(z) \psi_{\nu}(z) \\
& \quad=[2 \pi \Gamma(-i \mu) \Gamma(i \nu)]^{-1} \int_{-\infty}^{\infty} d z \exp \left(\frac{z}{2}\right) K_{-i \mu}\left[A \exp \left(\frac{z}{2}\right)\right] K_{i \nu}\left[A \exp \left(\frac{z}{2}\right)\right] \\
& =\pi[4 A \Gamma(-i \mu) \Gamma(i \nu)]^{-1}\left\{\cosh \left[\frac{\pi}{2}(\mu+\nu)\right] \cosh \left[\frac{\pi}{2}(\mu-\nu)\right]\right\}^{-1}
\end{aligned}
$$

To be able to describe really the classically evolving Universe, the packet (25) (26) should be sufficiently narrow, i.e. $c_{1}\left(\nu_{1}, \nu_{2}, \varrho\right)$ and $c_{2}\left(E_{k}\right)$ should not deviate from zero values beyond a small vicinity of their arguments near $\left(\bar{\nu}_{1}, \bar{\nu}_{2}, \bar{\varrho}\right)$ and $\bar{E}_{k}$. Therefore,

$$
\mu+\nu \approx 2 \bar{\nu} ; \quad \mu-\nu \approx \frac{A \omega}{2 \bar{\nu}}
$$

where $\omega=A^{-1}\left(\mu^{2}-\nu^{2}\right)$ is the difference between the two values of the parameter $E_{k}$ corresponding to the quantum numbers $\mu$ and $\nu$. Note that the matrix element (27) depends weakly on $\nu$ and is sharply decreasing when $|\mu-\nu|$ increasing. And so, making use of the approximations (28) one obtaines, for the average exponents $\exp (z / 2)$

$$
\begin{aligned}
\overline{\exp \left(\frac{z}{2}\right)} & =\frac{1}{4} \tanh (\pi \bar{\nu}) \int_{-\infty}^{\infty} d \omega \frac{\exp \left[-i \omega\left(t-t_{0}\right)\right]}{\cosh \left(\frac{\pi A \omega}{4 \bar{\nu}}\right)} \\
& =\frac{\bar{\nu} \tanh (\pi \bar{\nu})}{A \cosh \left[\left(2 A^{-1} \bar{\nu}\left(t-t_{0}\right)\right]\right.} .
\end{aligned}
$$

In the classical limit $\bar{\nu}$ is large, hence $\tanh (\pi \bar{\nu}) \approx 1$, and, comparing (29) with the classical expression (22), one concludes that

$$
\alpha=\frac{\bar{\nu}_{2}}{A_{2}}=\frac{3}{8} \bar{\nu}_{2} ; \beta=\frac{\bar{\nu}_{1}}{A_{1}}=\frac{3}{2} \bar{\nu}_{1} .
$$

From the (25), the equation for the mean values follows $(k=0)$ :

$$
\frac{3}{2} \bar{\nu}_{1}^{2}-\frac{3}{8} \bar{\nu}_{2}^{2}+\frac{1}{2} \bar{\varrho}^{2}=\bar{E},
$$

because $\overline{\nu_{1}^{2}} \approx \bar{\nu}_{1}^{2}$ an so on. Comparison of (23) and (30) gives

$$
\bar{\varrho}=C_{s} .
$$

These results reveal the following possible cosmological scenarios for the BianchiIX universe at the semiclassical stage of its evolution according to a relation between the parameters $\bar{E}$ and $C_{s}$ of the two condensates. 
1. Empty space $\left(C_{s}=0, \bar{E}_{k}=0\right) ; \alpha=\frac{\beta}{2}$.

In the limit $t= \pm \infty$

$$
a^{2}=b^{2}=\frac{\beta}{8} ; c^{2}=0,
$$

i.e. the metric (3) asymptotically takes the form

$$
\begin{gathered}
d s^{2}=\left(\beta c^{2}\right) d t^{2}-\frac{\beta}{8}\left(d \vartheta^{2}+\sin ^{2} \vartheta d \varphi^{2}\right), \\
\vartheta=x^{1}, \quad \varphi=x^{2} .
\end{gathered}
$$

When reaching singularity in one of the dimensions, two others form a stationary space of constant curvature. Here one deals with a regime of dynamical compactification, a space with simple topology being compactified.

2. Space is filled with the medium having positive energy density: $\bar{E}_{k}<$ $\frac{1}{2} C_{s}^{2} ; \alpha>\frac{1}{2} \beta$.

For $\alpha=\beta, t_{0}=0$ the model is isotropic.

For $\alpha=\beta, t_{0} \neq 0$ the model is unisotropic, but the singularity has an isotropic nature.

For $\frac{1}{2} \beta<\alpha<\beta$ in the pre-singular state $a^{2}=b^{2} \gg c^{2}$, i.e. $(2+1)$-dimensional space-time arises, where the 2 -space has constant curvature.

For $\alpha>\beta$ in the pre-singular state $c^{2} \gg a^{2}=b^{2}$, however, the model is not reduced to the space of less dimensions.

In all the cases for $\bar{E}_{k}<\frac{1}{2} C_{s}^{2}$ space at singularity is contracted to a point.

3. Space is filled with the medium having negative energy density: $\bar{E}_{k}>$ $\frac{1}{2} C_{s}^{2} ; \alpha<\frac{1}{2} \beta$.

At $t= \pm \infty$ the third space dimension is compactified $\left(c^{2} \rightarrow 0\right)$, and the remaining two-dimensional space of constant curvature is infinitely expanding. In the special case $\alpha=\beta / 4$ the scale factor $a=b$ is increasing exponentially in proper time.

So, the GVC, affecting coupling between the constants $\alpha$ and $\beta$ through the controlling parameter $\bar{E}_{k}$, determines a cosmological scenario which may contain the following phenomena:

- cosmological expansion and contraction of space;

- cosmological singularity;

- compactification of space dimensions;

- asymptotically stationary space of less dimensions;

- inflation of the Universe.

One can see that even such a simplified model reveals a number of effects probable from the standpoint of the modern cosmological ideas. Introduction of the GVC to the theory enlarges the number of possible cosmological scenarios, a concrete value of the parameter $E_{k}$ being formed at the quantum stage of the Universe existence.

Evidently, every classical cosmological evolution scenario must be in correspondence with some configuration of the wave packet (25) - (26). But not all the 
solutions to the SE describe classical universes, i.e. form sufficiently narrow packets to satisfy the conditions (28); in addition, even those wave packets, for which a transition to the semiclassical regime is possible, may prove to be unstable. Therefore in our approach the known problem of initial conditions for classical evolution is formulated as the problem of choice of the Universe quantum state. A quantum state in the Bianchi-IX model is determined by a concrete kind of the function

$$
\tilde{C}\left(\nu_{1}, \nu_{2}, \varrho, E_{k}\right)=c_{2}\left(E_{k}\right) c_{1}\left(\nu_{1}, \nu_{2}, \varrho\right)
$$

describing a wave packet structure.

We do not know the way the choice of the quantum state is being made by. Perhaps, it is realized according to statistical laws in the process of the Universe creation from "Nothing". In the next paper we intend to discuss the hypothesis according to which the act of the Universe creation is understood as, occurring out of time, quantum transition from the special singular state to one of the Universe physical states which the wave packets $(25)$ - (26) correspond to.

\section{Conception of Time}

In the previous section the transition to the semiclassical regime was considered in the GVC time. As a matter of fact, there exist four different concepts of time in the ordinary quantum theory:

- the Heisenberg time - the time parameter in Heisenberg representation; it is introduced equally with space coordinates by the device preparing an initial state of an object (preparator);

- the Schrödinger time introduced by the registrating device(registrator);

- the time in the interaction representation defined by preparators and registrators in the asymptotical regions of space-time;

- the world time defined on the object itself in the semiclassical regime (semiclassical proper time).

In QGD the only clock carrier is a GVC, and the question about availability of the Heisenberg time is closely related to the question about the Universe creation. As to the semiclassical proper time (SPT), its existence is not predetermined by the theory for, as it was mentioned above, a semiclassical regime is not predetermined itself. But, having such a regime actually, let us see how to deal with the SPT in the present theory.

From the very beginning we should emphasize that the SPT has nothing to do with the GVC as the OM carrier. By transition to the semiclassical regime the integrity effects are weakening; as a result, the fact of the GVC existence shows only through the parameters responsible, in our case, for the wave packet satisfactory width. So, the SPT does not depend on the GVC time, and the question, whether we can co-ordinate our semiclassical clocks with the GVC ones, seems to be of considerable physical significance in view of obtaining information about the quantum stage of the Universe evolution. 
A semiclassical wave packet may also be presented in the form

$$
\begin{aligned}
\Psi_{k}\left(z_{1}, z_{2}, \phi, t\right) & =\iint_{-\infty}^{\infty} d E_{k} d \varrho d \nu_{1} d \nu_{2} \tilde{C}\left(\nu_{1}, \nu_{2}, \varrho, E_{k}\right) \\
& \times \exp \left[-i E_{k}\left(t-t_{0}\right)+i \sigma_{1}\left(z_{1}\right)+i \sigma_{2}\left(z_{2}\right)\right] \\
& \times \psi_{\varrho}(\phi) \delta\left(\frac{3}{2} \nu_{1}^{2}-\frac{3}{8} \nu_{2}^{2}+\frac{1}{2} \varrho^{2}-E_{k}\right) .
\end{aligned}
$$

Here the sum $\sigma_{1}\left(z_{1}\right)+\sigma_{2}\left(z_{2}\right)$ is the part of the classical action $S\left(z_{1}, z_{2}, \phi, t\right)$, determining its dependence on $z_{1}$ and $z_{2}$ (the scalar field is treated as essentially quantum). The functions $\sigma(z)$ satisfy the equations

$$
\frac{\partial \sigma}{\partial z}=\sqrt{\frac{\bar{\nu}^{2}}{4}-\frac{A^{2}}{4} \exp (z)} .
$$

Knowing the dependence of the classical action on the variables $z$, one can reconstruct the evolution law (22) with the help of the standard procedure. But the two mentioned methods of going over to the classical limit are applicable owing to the explicit dependence of the GS on time. And this, in turn, is caused by the available, in the theory, indication of the concrete choice of an RS which the time $t$ is measured in.

In the classical limit a classical subsystem of the physical object itself can be considered as an RS. Such a subsystem cannot fill the whole space; it is admissible that it would occupy a limited region of space. So, we will refer to such an RS as to a local one. The appearance of the time $\tau$, introduced as a parameter along a classical path, is associated with that RS.

A derivative with respect to path length can be defined by the following way:

$$
\frac{d}{d t}=u(\tau) \nabla S \nabla
$$

where $u(\tau)$ is an arbitrary function, $\nabla S$ is a tangent vector to the path;

$$
\nabla=\left(2 \sqrt{3} \frac{\partial}{\partial z_{1}}, \quad i \sqrt{3} \frac{\partial}{\partial z_{2}}\right)
$$

On the other hand,

$$
\frac{d}{d \tau}=\frac{d z_{1}}{d \tau} \frac{\partial}{\partial z_{1}}+\frac{d z_{2}}{d \tau} \frac{\partial}{\partial z_{2}}
$$

whence

$$
\frac{d z_{1}}{d \tau}=12 u(\tau) \frac{d \sigma_{1}}{d z_{1}} ; \quad \frac{d z_{2}}{d \tau}=-3 u(\tau) \frac{d \sigma_{2}}{d z_{2}} .
$$

From (31) one obtaines

$$
\frac{d z_{1}}{d \tau}=4 u(\tau) \sqrt{\beta^{2}-\exp \left(z_{1}\right)} ; \quad \frac{d z_{2}}{d \tau}=-4 u(\tau) \sqrt{\alpha^{2}-\exp \left(z_{2}\right)},
$$


and, in the result of integration,

$$
\begin{gathered}
\exp \left(\frac{z_{1}}{2}\right)=\beta \cosh ^{-1}\left(2 \beta\left[\tilde{u}(\tau)-\tau_{0}^{\prime}\right]\right) ; \exp \left(\frac{z_{2}}{2}\right)=\alpha \cosh ^{-1}\left(2 \alpha\left[\tilde{u}(\tau)-\tau_{0}\right]\right) \\
\tilde{u}(\tau)=\int u(\tau) d \tau ; \quad \tau_{0}, \tau_{0}^{\prime}=\text { const. }
\end{gathered}
$$

The time $\tau$ of a local observer emerges irrespectively of the time $t$ existence, but both the times may correlate between them. To bring the expressions 222 and (32) in correspondence, it is sufficient to put $u(\tau)=1$. This act assumes the following operations:

(i) determining the rate of the Universe evolution by means of cosmological observations;

(ii) fixing, on this data base, the GVC parameters and the state equation of it;

(iii) reconstruction of the gauge from the state equation and so fixing the GVC time scale.

In the ordinary quantum mechanics the time variable involving in SE may fail to coincide with that appearing in Heisenberg operator equations, as well as with the world time in which semiclassical system dynamics can be described. In the quantum mechanics the hypothesis about equivalence of the mentioned time variables is used, though this is nowhere specified. In the considered example we have manifested that the time variables used for describing a quantum system evolution are different in general. In QGD times associated with different observers can be brought to agreement with each other by choice of a gauge condition.

\section{References}

[1] V. A. Savchenko, T. P. Shestakova and G. M. Vereshkov, Int. J. Mod. Phys. A11,4473 (1999), gr-qc/9909047.

[2] E. S. Fradkin and G. A. Vilkovisky, Phys. Lett. B55, 224 (1975).

[3] I. A. Batalin and G. A. Vilkovisky, Phys. Lett. B69, 309 (1977).

[4] E. S. Fradkin and G. A. Vilkovisky, CERN Report No TH-2332, 1977.

[5] E. S. Fradkin and T. E. Fradkina, Phys. Lett. B72, 343 (1978).

[6] I. A. Batalin and E. S. Fradkin, in Group Theoretical Methods in Physics (Moscow, 1980), Vol. II.

[7] C. Teitelboim, Phys. Rev. D25, 3159 (1982); D28, 297 (1983); D28, 310 (1983).

[8] J. J. Halliwell, Phys. Rev. D38, 2468 (1988).

[9] J. J. Halliwell and J. B. Hartle, Phys. Rev. D43, 1170 (1991).

[10] S. W. Hawking and J. C. Luttrell, Phys. Lett. B143, 83 (1984).

[11] D. Marolf, Class. Quant. Grav. 12, 1441 (1995).

[12] S. Chakraborty, Int. J. Theor. Phys. 30, 849 (1991).

[13] R. Paternoga, R. Graham, Phys. Rev. D54, 4805 (1996).

[14] J. Louko, Phys. Rev. D51, 586 (1995).

[15] H. Kodama, Prog. Theor. Phys. 80, 1024 (1988).

[16] R. Graham, Phys. Rev. D48, 1602 (1993). 
[17] R. Graham and H. Luckock, Phys. Rev. D49, 4981 (1994).

[18] A. D. Y. Cheng, P. D. D'Eath and P. R. L. V. Moniz Phys. Rev. D49, 5246 (1994).

[19] A. D. Y. Cheng, P. D. D'Eath, Class. Quant. Grav. 13, 3151 (1996).

[20] P. V. Moniz, Int. J. Mod. Phys. A11, 1763 (1996).

[21] J. E. Lidsey, Phys. Rev. D49, 599 (1994).

[22] B.S. DeWitt, Phys. Rev. 160, 1113 (1967).

[23] L.D. Landau and E.M. Lifshitz, Theory of Fields (Nauka, Moscow, 1988). 